\title{
Steady-state Serrated Deformation of Metallic Glass during Indentation
}

\author{
K.Y.Ng ${ }^{1}$, L. Zuo ${ }^{1,2}$, A.H.W. Ngan ${ }^{1, *}$ \\ ${ }^{1}$ Department of Mechanical Engineering, The University of Hong Kong, Pokfulam Road, \\ Hong Kong, PR China \\ ${ }^{2}$ Now at School of Molecular and Microbial Sciences, The University of Queensland, St. \\ Lucia, Brisbane, QLD 4067, Australia \\ * Correspondence Author (Email: hwngan@hku.hk)
}

\begin{abstract}
Using an exponential load profile with a Berkovich tip, the strain bursts produced during nanoindentation of a $\left(\mathrm{Cu}_{55} \mathrm{Mg}_{33} \mathrm{Y}_{12}\right)$-Be bulk metallic glass are found to reach a steady state, in which the hardness becomes constant, the displacement jumps normalized by the indent depth approach a constant range $(1.4 \pm 1.2) \%$ and correspond to hardness reduction by $(2.8 \pm 2.4) \%$, and the inter-burst duration multiplied by the strain rate approaches a constant range $1.8 \pm 0.6 \%$. This steady-state behavior is insensitive to the strain rate used.
\end{abstract}

Keywords: Nanoindentation; strain rate; plastic deformation; bulk metallic glass (BMG) 


\section{Introduction}

There is increasing evidence that plastic deformation in a wide range of materials is in the form of small avalanches or strain bursts. De Hosson et al. [1] used nuclear magnetic resonance to study dislocation dynamics in Al-Zn alloys and found that moving dislocations can advance over a broad spectrum of distances. Weiss, Miguel and coworkers [2-6] measured the acoustic emissions during creep deformation of ice single crystals and found that the emissions followed a power-law distribution characteristic of self-organized critical behavior [7-8]. Nanoindentation experiments on a wide range of materials also revealed displacement bursts of nanometric sizes during deformation [9], and in particular, nanoindentation revealed serrated deformation in bulk metallic glasses [10-17].

Because the occurrence of strain bursts is in general stochastic, their quantification is not a trivial matter. The usual tensile or compression tests are not ideal because the same mass of material is being strained, and so the strain bursts emitted at a late stage of deformation will be not under the same conditions as those emitted at the beginning of the straining, especially if the deformation microstructure evolves in a systematic manner with strain. In this paper, we used indentation with a self-similar (i.e. Berkovich) indenter to study the deformation bursts in a copper-based bulk metallic glass. At steady state, the deformation front in this case propagates continuously outward at a rate which maintains self similarity with the penetration of the indenter, and a condition can be achieved in which the material behavior probed at a late stage of the experiment is comparable to that probed at an early stage. As shown by Lucas and Oliver [18], this steady state is achieved with a loading profile which results in the indentation strain rate being constant during the indentation.

\section{Experimental}

A $\left(\mathrm{Cu}_{55} \mathrm{Mg}_{33} \mathrm{Y}_{12}\right)-\mathrm{Be}(\mathrm{wt} \%)$ bulk metallic glass rod $4 \mathrm{~mm}$ in diameter was supplied by Dr. M.G. Wang of Northeastern University, Shenyang, P.R. China. This was sectioned and then embedded using an epoxy resin into a matching $5 \mathrm{~mm}$ hole at the 
center of a $50 \mathrm{~mm}$ diameter brass cylinder block. The embedded assembly was then mechanically polished followed by electro-polishing at $15 \mathrm{~V}$ in a solution of $25 \% \mathrm{v} / \mathrm{v}$ perchloric acid ( $70 \%$ concentrated) in glacial acetic acid, to achieve a surface smoothness of smaller than $1 \mu \mathrm{m}$. X-ray diffraction (not shown) revealed no crystalline peak, indicating that the sample's structure was amorphous. The $\mathrm{Cu}_{55} \mathrm{Mg}_{33} \mathrm{Y}_{12}$ composition of the sample was determined by Electron Dispersive X-ray Analysis.

Nanoindentation experiments were conducted using a CSEM Nanohardness Tester. An exponential loading profile $P(t)=P_{0} \exp (K t)$, where $P_{0}$ is a small pre-set load and $K$ is a constant, was used, and as shown by Lucas and Oliver [18], this loading profile leads to a constant-strain-rate condition when a self-similar indenter is used. Under this condition, $P \propto h^{2}$, where $h$ is indentation depth, and with a self-similar indenter, the indentation strain rate is $\dot{\varepsilon}_{I}=\dot{h} / h=K / 2$ [18]. $\dot{\varepsilon}_{I}$ here is actually a scaling factor in the strain-rate field [19], and hence it may be conceived as an effective strain rate inside the deforming volume [18]. In the present experiment, the pre-load was set to be $P_{0}=0.5 \mathrm{mN}$, and three values of $K$, namely, $0.001151,0.01151$ and $0.1151 \mathrm{~s}^{-1}$, corresponding to $\dot{\varepsilon}_{I}$ of $5 \times 10^{-4} \mathrm{~s}^{-1}, 5 \times 10^{-3} \mathrm{~s}^{-1}$ and $5 \times 10^{-2} \mathrm{~s}^{-1}$, were used. At each strain rate, typically 20-25 indentation runs, corresponding to typically 850 bursts, were performed under similar conditions to produce an ensemble of data for statistical analysis.

Fig. 1 shows a typical section of the indenter displacement vs time graph, which reveals that the deformation of the metallic glass sample is jumpy. The raw displacementtime data (after thermal drift correction) consists of a high-frequency signal of amplitude $\sim 1.3 \mathrm{~nm}$, superimposed on a low-frequency signal of much larger amplitudes. The lowfrequency signal is evidently the real displacement bursts representing the jumpy plastic deformation of the metallic glass, and the high-frequency, low-amplitude signal is the noise floor of the machine. In a previous paper [9], we have developed a simple protocol to identify the bursts, and to distinguish them from the noise. In this work, we have modified this protocol to speed up the burst identification process. In brief, three stages are used to identify the bursts. The first two stages involve a data-smoothing algorithm for filtering out the noise but at the same time preserving the original shape of the curve. The last stage involves a burst search algorithm to detect and record the burst data throughout the whole displacement-time curve according to the preset values, without the 
need to curve-fit the raw data. The first stage of smoothing makes use of a maximum homogeneity smoothing technique [20-21], and the second state employs a median smoothing technique [22]. In the burst search algorithm, the slope $\mathrm{d} y / \mathrm{d} x$ between each neighboring data pair is first normalized by the $y_{o} / x_{o}$ value, where $y_{o}\left(x_{o}\right)$ is the maximum $y$-value in the smoothened $y(x)$ data curve, to yield a dimensionless quantity $\xi=(\mathrm{d} y / \mathrm{d} x) /$ $\left(y_{o} / x_{o}\right)$. A threshold value $\xi_{c}$ for $\xi$ is pre-defined, and the algorithm detects the existence of a potential burst whenever $\xi$ for any data pair is larger than $\xi_{c}$. Since a real burst may last over a few pairs of consecutive data points, the data points for which the condition $\xi$ $>\xi_{c}$ has just started and ended in a series are recorded respectively as the beginning and ending points of a burst. In the present investigation, typical values for $\xi_{c}$ chosen were from 15 to 80 . Fig. 1 shows the bursts identified from the raw data using this algorithm with $\xi_{c}=15$. Comparing with our previous protocol [9], both methods can identify the locations of all the expected strain bursts in a noisy situation, but the new protocol works much faster.

\section{Results}

The displacement-time graphs under all conditions tested are jumpy as shown in Fig. 1. Fig. 2(a) shows plots of $P / h^{2}$ vs $h$ for typical runs of the nanoindentation under the three strain rates used. It can be seen that for indent depths larger than about $300 \mathrm{~nm}$, $P / h^{2}$ tends to a constant of about $70 \mathrm{GPa}$. This indicates that (i) the self-similar, steadystate constant-strain-rate condition [18] is approximately reached for the later part of the indentation, and (ii) the hardness, which is proportional to $P / h^{2}$, approaches a steadystate value which is approximately independent of the strain rate. In other words, the strain-rate sensitivity of strength of the metallic glass is very small within the range studied.

The displacement bursts $\Delta h$ are not of similar sizes at different stages of the indentation, but they become larger as the indent depth $h$ increases. However, the dimensionless ratio $\Delta h / h$ does not exhibit an increase in scatter on increasing $h$, as shown in Fig. 2(b). Fig. 2(b) shows that the scatter in the $\Delta h / h$ data becomes rather steady as $h$ is larger than $300 \mathrm{~nm}$ or so, and this is also the range when the hardness 
$P / h^{2}$ becomes steady in Fig. 2(a). The average value of $\Delta h / h$ also exhibits only a very weak sensitivity on strain rate - it is about $1 \%$ for the two lower values of $K$, and this increases to about $1.5 \%$ for the highest value of $K$.

As Fig. 1 shows, the deformation of the present metallic glass comprises clearly identifiable bursts, the occurrence of which is sequential but stochastic. In each experiment, the bursts generated can be counted by the order $m$ in which they occur, i.e. the first burst has $m=1$, the second has $m=2$, and so on. In the ensemble of repeated runs of indentation experiment under the same conditions (i.e. the same strain rate), statistics on how synchronized the emission of bursts is can be obtained by considering the fraction $F_{m}$ of experiments in the ensemble in which the $m^{\text {th }}$ burst has not occurred by time t. $F_{m}$ can be obtained from the processed burst data shown in Fig. 1 as follows. The time $t$ at which the $m^{\text {th }}$ burst occurred in each run of the experiment is recorded. The whole ensemble of repeated experiments then generates different times $t$ at which the $m^{\text {th }}$ burst occurred, and these times are then ranked into ascending order. $F_{m}$ is then assigned the value $F_{m}=1-i / M$, where $i$ is the rank of each time value, and $M$ is the number of experiments in the ensemble. $F_{m}$ here is the fraction of the $M$ experiments in which the $m^{\text {th }}$ burst occurs later than the time corresponding to rank $i$. Fig. 3(a) show the $F_{m}(t)$ plots for the indentation rate $K=0.01151 \mathrm{~s}^{-1}$, where the time $t$ is shown in a normalized scale $K t / 2$. By comparing with similar plots for the other two indentation rates used (not shown), the variations of the $F_{m}$ vs $K t / 2$ plots for the three different strain rates are very small, i.e. the burst emission statistics are also rather insensitive to strain rate, within the range studied. It can also be seen from Fig. 3(a) that the $F_{m}$ curves become more equispaced for the later bursts. The time $t_{m}$ at which $F_{m}\left(t_{m}\right)=0.5$ is the average time at which the $m^{\text {th }}$ burst occurred, and so the time interval $\delta t_{m}=t_{m}-t_{m-1}$ between two successive bursts is a measure of the average time lapse between the two bursts. Fig. 3(b) shows the $K \delta t_{m} / 2$ values evaluated from the $F_{m}$ vs $K t / 2$ plots, and it can be seen that the value $K \delta t_{m} / 2$ settles into an asymptotic value of about 1 to $2 \%$, for later bursts with high $m$ values, irrespective of the strain rate used. 


\section{Discussion}

Serrated or inhomogeneous deformation during nanoindentation has been observed in different bulk metallic glasses including Pd-based [10, 12, 13, 14, 15, 16], La-based [11, 15], Zr-based [14, 15, 17], Cu-based [15] and Mg-based [16] glasses, at low indentation rates and at temperatures well below glass transition. At high indentation rates, deformation tends to be homogeneous $[13,14,16]$. Unfortunately, these experiments employed either constant loading rate or indenter velocity, which are not conditions that would trigger constant strain rate or steady state in the indentation process. The indentation strain rate $\dot{\varepsilon}_{I}=\dot{h} / h$ used, although not in steady state, was reported in a few studies by Schuh and Nieh, in La-25Al-10Cu-5Ni-5Co [11], Pd-40Ni-20P [12], Pd$30 \mathrm{Cu}-10 \mathrm{Ni}-20 \mathrm{P}$ [14] and $\mathrm{Zr}-10 \mathrm{Al}-1-\mathrm{Ni}-15 \mathrm{Cu}$ [14] glasses. Their results indicate that, in

these metallic glasses, deformation tends to transit to the homogeneous regime for $\dot{\varepsilon}_{I}>$ $10^{0}$ to $10^{1} \mathrm{~s}^{-1}$. The strain rate used in the present study is from $5 \times 10^{-4} \mathrm{~s}^{-1}$ to $5 \times 10^{-2} \mathrm{~s}^{-1}$, and so the serrated deformation observed in the present $\left(\mathrm{Cu}_{55} \mathrm{Mg}_{33} \mathrm{Y}_{12}\right)$-Be glass is well expected.

To our best knowledge, the present study is the first to study the constant-strainrate, steady-state indentation behavior of a metallic glass. An ensemble approach is also used to enable proper statistical analysis of the flow serrations or bursts. The present results reveal two interesting features of such a steady state, namely, (i) the normalized displacement burst $\Delta h / h$ in Fig. 2(b) fluctuates within a constant window of $1.4 \pm 1.2 \%$ on increasing $h$, and, (ii) the normalized inter-burst duration $K \delta t_{m} / 2$ in Fig. 3(b) also fluctuates within a constant window of $1.8 \pm 0.6 \%$ for the later bursts. The steady-state range of $\Delta h / h$ is smaller but comparable to that of $K \delta t_{m} / 2$, and both are independent of strain rate.

Hardness $H$ is defined as the indentation load divided by the indent area, and at steady-state condition, it is given by

$$
H=c \frac{P}{h^{2}}
$$


where $c$ is a constant for a specific indenter. The present experiments are load-controlled, and so during a burst in which the indenter displacement jumps by $\Delta h, P$ is approximately constant. The jump $\Delta h$ is due to sudden softening of the material corresponding to a reduction in its hardness $\Delta H$ given by

$$
-\frac{\Delta H}{H}=2 \frac{\Delta h}{h} \text {. }
$$

Since the hardness $H$ is constant in the steady-state (Fig. 2(a)), a constant value of $\Delta h / h$ means that the hardness drop $\Delta H$ due to softening is invariant along the indentation path. Hence, strain bursts emitted in a self-similar, steady-state indentation situation produce a consistent hardness drop $\Delta H$, and since $\Delta h / h=(1.4 \pm 1.2) \%$, this hardness drop is about $(2.8 \pm 2.4) \%$. The parameter $\Delta h / h$ may also be regarded as a measure of the strain of each burst. That the softening $\Delta H$ and the serration strain magnitude $\Delta h / h$ are statistically invariant along the indentation path is an important feature of steady-state indentation not noted before by previous researchers.

The reason why $K \delta t_{m} / 2$ is comparable to $\Delta h / h$ may be understood from the schematic of the serrated deformation shown in the inset in Fig. 1. Here, $\delta t$ is the time interval between two successive bursts, and $\delta h$ is the rise in the mean-field displacement during $\delta t$. If the displacement bursts $\Delta h$ constitute a significant portion of the meanfield displacement, then we expect

$$
\Delta h \widetilde{<} \delta h .
$$

At steady state with a load profile $P(t)=P_{0} \exp (K t)$, the mean-field displacement will be $h=h_{0} \exp (K t / 2)$ where $h_{0}$ the an initial displacement corresponding to $P_{0}$ [18]. Therefore, during the small time interval $\delta t$ between two bursts, the mean-field displacement rises by $\delta h=h K \delta t / 2$, and from eqn. (3), we have

$$
\Delta h / h \widetilde{<} K \delta t / 2 .
$$

We thus expect that if $\Delta h / h$ settles into a steady-state range corresponding to an invariant hardness drop $\Delta H$, the quantity $K \delta t / 2$ also settles into a similar (but lower) steady-state range according to eqn. (4), with repercussions on the spacing between the $F_{m}$ curves in Fig. 3. The results in Figs. 2 and 3 are thus intricately related. 
The present results confirm that the strain bursts emitted during steady-state indentation of metallic glass exhibit a mean-field size, and are not of a scale-free, powerlaw distributed type as reported in other deformation conditions [2-8, 24-25]. A similar observation was made in a previous study [9] on a $\left(\mathrm{Zr}_{63} \mathrm{Ni}_{10} \mathrm{Cu}_{16} \mathrm{Al}_{11}\right)$-Be bulk metallic glass, as well as on other materials, in the indentation situation. As mentioned before, the deformation front during indentation with a sharp indenter is propagating continuously outward so that at each increment of the penetration of the indenter, a fresh part of the specimen joins the deformation zone. Under suitable conditions, this may allow a selfsimilar, steady state to be reached in which a later part of the indentation process is comparable to an initial part. On the contrary, in ordinary tensile or compression tests, the same material volume is strained and if the deformation microstructure evolves rapidly with strain, superposing different stages of the deformation in an analytical process may obscure the effects of any individual mean-field spatial or temporal scale of the microstructure at each stage of the deformation, producing apparently scale-free overall statistics. Further work is required to understand more fully the statistical behavior of material deformation.

\section{Conclusions}

Nanoindentation on a $\left(\mathrm{Cu}_{55} \mathrm{Mg}_{33} \mathrm{Y}_{12}\right)$-Be bulk metallic glass reveals jerky deformation behavior at room temperature. Using an exponential load profile with a Berkovich tip leads to a steady state condition in which the hardness, the displacement burst normalized by the instantaneous displacement, and the inter-burst duration multiplied by the strain rate used, are all statistically invariant on increasing indentation depth. The steady-state ranges of these parameters are also found to be insensitive to the

strain rates used in the experiments, which vary by a factor of 100 from $5 \times 10^{-4} \mathrm{~s}^{-1}$ to $5 \times 10^{-2} \mathrm{~s}^{-1}$. These results indicate that the softening associated with each burst produces a reduction in hardness within the range of $(2.8 \pm 2.4) \%$, and that the strain in each burst fluctuates within the range $(1.4 \pm 1.2) \%$, and these are rate insensitive. The protocol used in this work is suitable for studying jerky deformation in general. 


\section{Acknowledgments}

The work described in this paper was supported by a grant from the Research Grants Council of the Hong Kong Special Administration Region, P.R. China (Project No. HKU7156/08E). The authors are grateful to Dr. M.G. Wang for supplying the BMG sample.

\section{References}

1. J.Th.M. De Hosson, G. Boom, U. Schlagowski, and O. Kanert, Acta Metall. 34 (1986) 1571

2. M.-C. Miguel, A. Vespingnani, S. Zapperi, J. Weiss, and J.-R. Grasso, Nature $410(2001) 667$

3. Weiss J, Miguel MC, Mater Sci Eng. A. 387(2004)292

4. Weiss J, Grasso JR, Miguel MC. Mater Sci Eng. A. 309 (2001)360

5. Miguel MC, Vespignani A., Zapperi S, Mater Sci Eng A. 309(2001)324

6. Miguel MC, Moretti P, Zaiser M, Zapperi S. Mater Sci Eng A 400(2005)191

7. P. Bak, C. Tang, and K. Wiesenfeld,Phys. Rev. Lett. 59(1987)381

8. P. Bak: How Nature Works (Springer-Verlag, Berlin, Germany, 1996.

9. Li H, A.H.W Ngan and Wang MG, J Mater Res. 20[11](2005)3072

10. Golovin YI, Ivogin VI, Khonik VA, Kitagawa K, Tyurin AI. Scirpta Mater. 45 (2001)947

11. T.G. Nieh, C. Schuh, J. Wadsworth and Y. Li, Intermetallics, 10(2002)1177

12. C.A Schuh, T.G. Nieh and Y. Kawamura, J. Mater. Res., 17(2002)1651

13. C.A. Schuh, A.S. Argon, T.G. Nieh and J. Wadsworth, Phil. Mag. 83(2003) 2585

14. C.A. Schuh and T.G. Nieh, Acta Mater., 51(2003)87

15. C.A. Schuh and T.G. Nieh, J. Mater. Res., 19(2004)46

16. C.A. Schuh, A.C. Lund and T.G. Nieh, Acta Mater. 52(2004)5879

17. P. Murali and U. Ramamurty, Acta Mater. 53(2005)1467

18. B.N. Lucas, W.C.Oliver, Metall Mater Trans A MATER TRANS A 30[3](1999)601

19. A.F. Bower, N.A. Fleck, A. Needleman, and N. Ogbonna, Proc. R. Soc. London, Ser. A 441(1993)97. 
20. M. Nago and T. Matsuyama, CVGIP 9(1979)394

21. F.Tomita and S. Tsuiji, IEEE-SMC 7(1977)107

22. J.W. Tukey: Exploratory Data Analysis, Addison-Wesley, Reading, Mass., 1971

23. A.H.W. Ngan and K.S. Ng: Transition from deterministic to stochastic deformation, submitted (2009).

24. D. M. Dimiduk, C. Woodward, R. LeSar, and M. D. Uchic, Science 312(2006)1188.

25. D.M. Dimiduk, M.D. Uchic, S.L. Rao, C. Woordward and T.A. Parthasarathy, Modelling Simul Mater Sci Eng 15(2007)135. 


\section{Figure Captions:}

Figure 1 - Typical section of the displacement-time graph during nanoindentation of the metallic glass. Inset shows schematic of serrated displacement-time graph (dash-dot line shows the mean-field displacement; thick solid line shows the actual displacement with serrations).

Figure 2 - (a) Plots of $P / h^{2}$ vs $h$ for the three strain rates. (b) Normalized magnitudes of bursts $\Delta h / h$ vs indentation depth $h$ at different strain rates.

Figure 3 - (a) Plots of $F_{m}$ vs normalized time $K t / 2$ for $K=0.01151 \mathrm{~s}^{-1}$. (b) Average inter-burst duration $\delta t_{m}=t_{m}-t_{m-1}$ vs burst order $m . t_{m}$ is the time at which $F_{m}\left(t_{m}\right)=0.5$. 


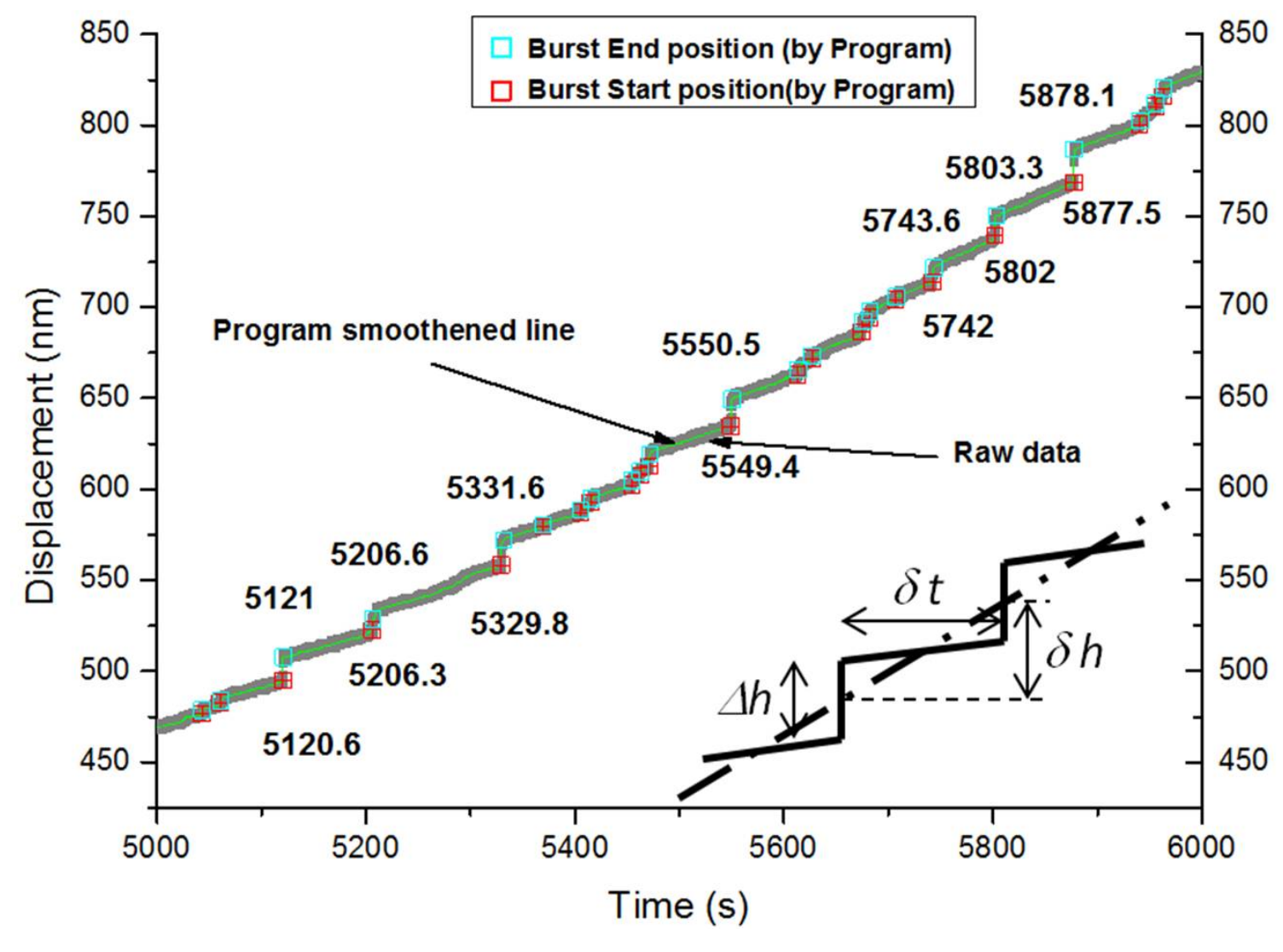

Figure 1 


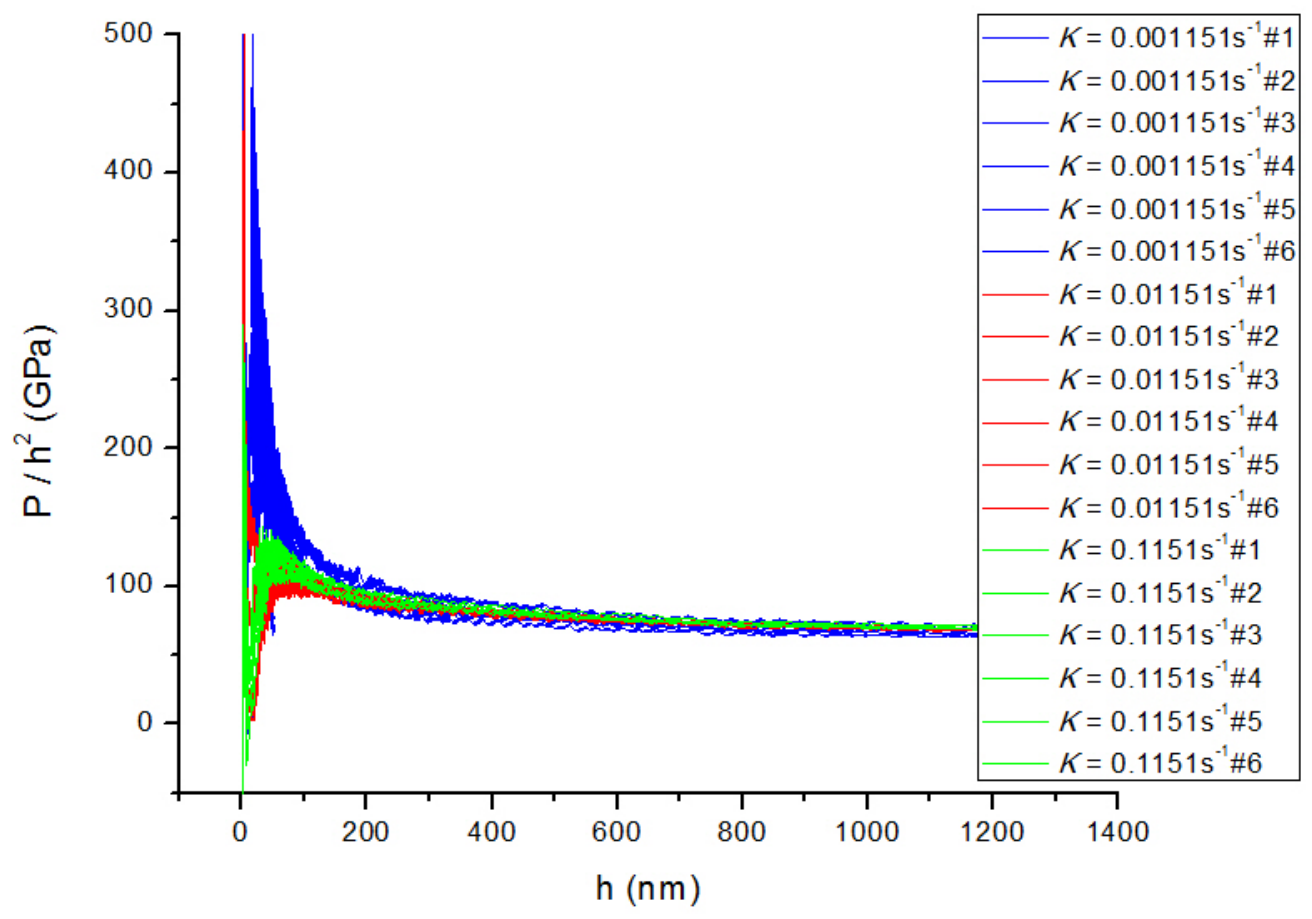

Figure 2 (a)

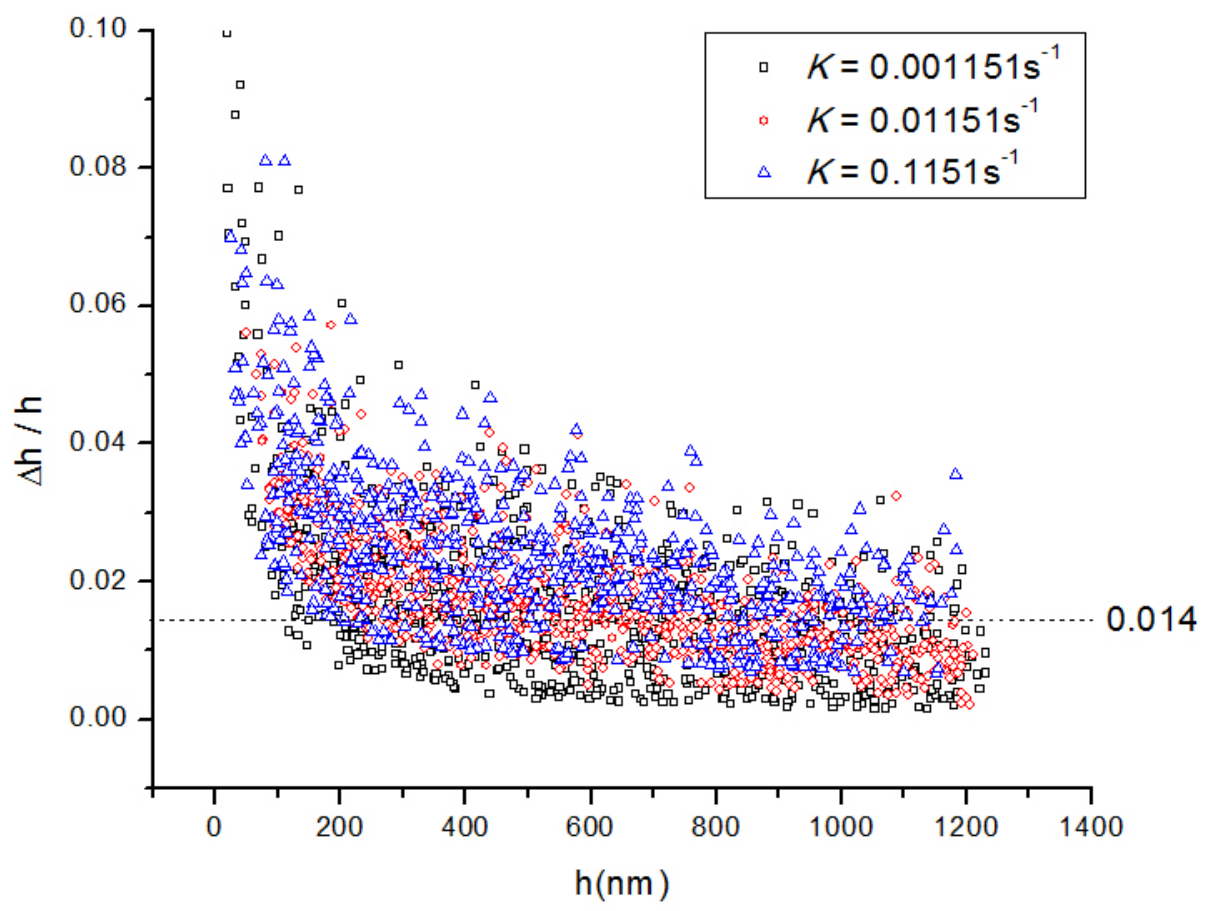

Figure 2 (b) 


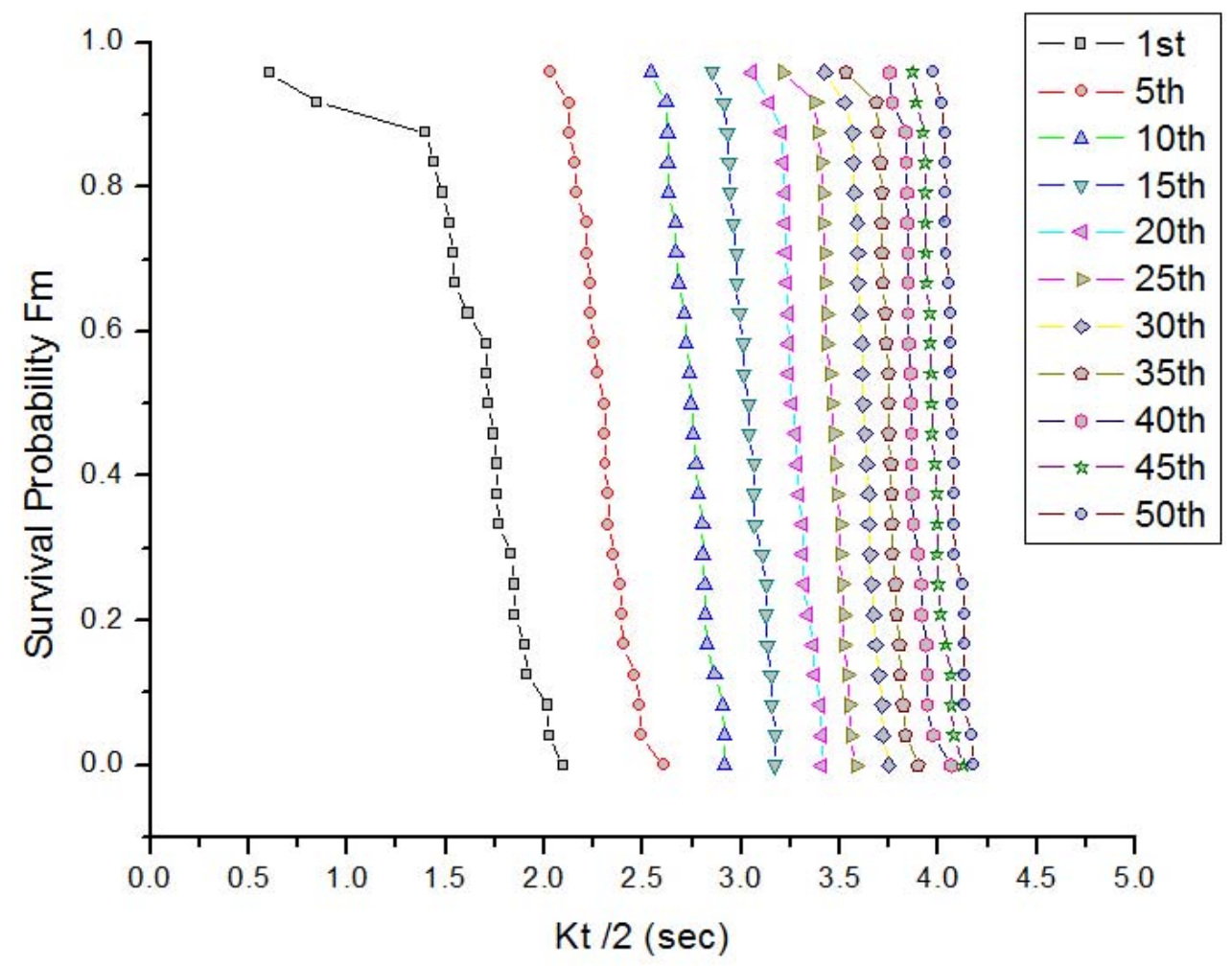

Figure 3 (a)

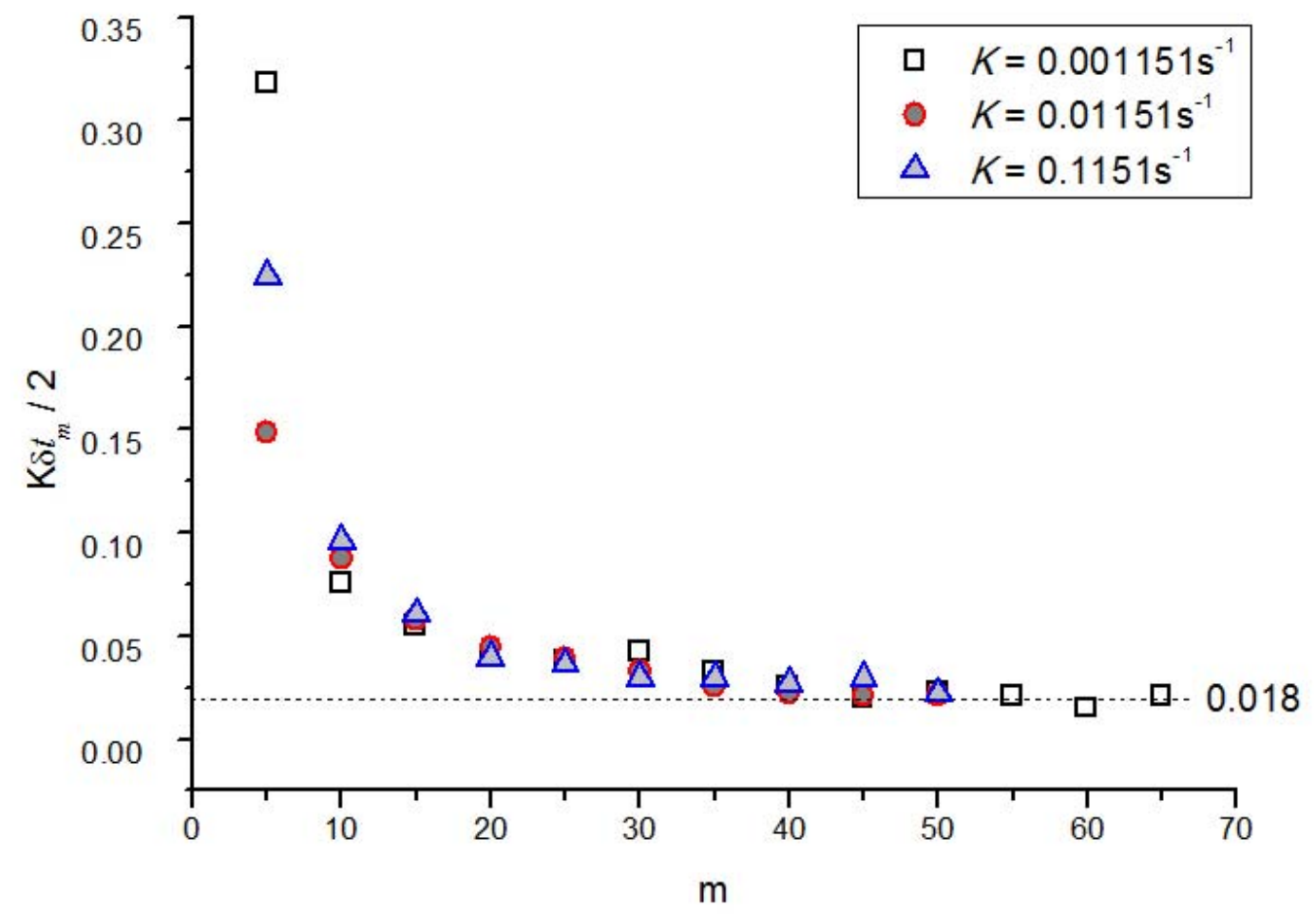

Figure 3 (b) 